\title{
Models of temporal enhanced ultrasound data for prostate cancer diagnosis: the impact of time-series order
}

Layan Nahlawi

Caroline Goncalves

Farhad Imani

Mena Gaed

Jose A. Gomez

Madeleine Moussa

Eli Gibson

Aaron Fenster

Aaron D. Ward

Purang Abolmaesumi

Parvin Mousavi

Hagit Shatkay 


\title{
Models of Temporal Enhanced Ultrasound Data for Prostate Cancer Diagnosis: The Impact of Time-Series Order
}

\author{
Layan Nahlawi ${ }^{\mathrm{a}}$, Caroline Goncalves ${ }^{\mathrm{a}}$, Farhad Imani ${ }^{\mathrm{b}}$, Mena Gaed ${ }^{\mathrm{c}}$, Jose A. Gomez ${ }^{\mathrm{c}}$, \\ Madeleine Moussa $^{\mathrm{c}}$, Eli Gibson ${ }^{\mathrm{d}}$, Aaron Fenster ${ }^{\mathrm{c}}$, Aaron D. Ward ${ }^{\mathrm{c}}$, Purang Abolmaesumi ${ }^{\mathrm{b}}$, \\ Parvin Mousavi*a, and Hagit Shatkay*a,e \\ aschool of Computing, Queen's University \\ ${ }^{b}$ Dept. of Electrical and Computer Engineering, University of British Columbia \\ ${ }^{\mathrm{c}}$ Dept. of Medical Biophysics, Pathology and Robarts Inst., Western University \\ ${ }^{\mathrm{d}}$ Centre for Medical Image Computing, University College London \\ ${ }^{\mathrm{e}}$ Dept. of Computer and Information Sciences, University of Delaware
}

\begin{abstract}
Recent studies have shown the value of Temporal Enhanced Ultrasound (TeUS) imaging for tissue characterization in transrectal ultrasound-guided prostate biopsies. Here, we present results of experiments designed to study the impact of temporal order of the data in TeUS signals. We assess the impact of variations in temporal order on the ability to automatically distinguish benign prostate-tissue from malignant tissue. We have previously used Hidden Markov Models (HMMs) to model TeUS data, as HMMs capture temporal order in time series. In the work presented here, we use HMMs to model malignant and benign tissues; the models are trained and tested on TeUS signals while introducing variation to their temporal order. We first model the signals in their original temporal order, followed by modeling the same signals under various time rearrangements. We compare the performance of these models for tissue characterization. Our results show that models trained over the original order-preserving signals perform statistically significantly better for distinguishing between malignant and benign tissues, than those trained on rearranged signals. The performance degrades as the amount of temporal-variation increases. Specifically, accuracy of tissue characterization decreases from $85 \%$ using models trained on original signals to $62 \%$ using models trained and tested on signals that are completely temporally-rearranged. These results indicate the importance of order in characterization of tissue malignancy from TeUS data.
\end{abstract}

Keywords: Hidden Markov Models, Time-series, Ultrasound, Prostate Cancer, Tissue Characterization, Temporal Order

\section{INTRODUCTION}

Prostate Cancer is highly prevalent, and is diagnosed in one in seven North American men during their lifetime. ${ }^{1,2}$ Diagnosis of prostate cancer requires measuring the prostate-specific antigen in the blood serum along with digital-rectal examination. If either test is abnormal, core needle biopsy of the prostate under Trans-Rectal UltraSound (TRUS) guidance is used for obtaining a definitive diagnosis. ${ }^{3}$ However, TRUS does not support patient-specific targeting and has low sensitivity for differentiation between indolent and aggressive cancer. ${ }^{4,5}$ Temporal enhanced ultrasound (TeUS) is a novel imaging technique that has been shown effective for tissue characterization in prostate cancer. ${ }^{6,7}$ TeUS is produced by sonicating tissue over a short period of time, without intentionally moving the tissue or the transducer. The result is a sequence (time series) of Radio Frequency $(\mathrm{RF})$ ultrasound frames. The pattern of change in echo-intensity of tissue along the time series varies from one tissue type to another. As such, TeUS carries tissue specific information. Using TeUS to augment biopsy procedures with maps of areas that are likely to be cancerous is a promising approach to overcome the limitations of TRUS-guided biopsies.

Hidden Markov Models (HMMs) are probabilistic models that capture order and pattern in time varying signals. $^{8}$ They are widely used in a variety of application domains such as speech recognition ${ }^{9}$ and DNA sequence

\footnotetext{
* These authors contributed equally to the work.
} 
alignment. ${ }^{10}$ We have previously used HMMs to model TeUS acquired from prostate tissue to successfully differentiate between malignant and benign tissue types within a tissue-characterization framework. ${ }^{11}$

Here we present new experiments, specifically aiming to investigate the impact of temporal order on the informative value of TeUS signals for tissue characterization. We assess the impact of changes in the order of RF frames over time on the performance of tissue characterization using HMMs, and show that order-variations indeed significantly affect performance. The rest of the paper is organized as follows: Section 2 presents our TeUS data along with our modeling approach; Section 3 demonstrates our tissue-characterization experiments and results; Section 4 summarizes the conclusions from this work.

\section{MATERIALS AND METHODS}

\subsection{Time-domain Representation of TeUS Data}

We acquired TeUS data from 9 patients who have undergone radical prostatectomy as part of prostate cancer treatment. The histopathology markings on the prostate slices of each patient are aligned with in vivo ultrasound images via a thorough registration process (see Imani et al. ${ }^{7}$ for details). Each patient is associated with a sequence of $128 \mathrm{RF}$ ultrasound frames, where each frame consists of $1276 \times 64$ intensity values. Each frame is divided into Regions of Interest (ROIs) of size $1.7 \times 1.7 \mathrm{~mm}$, where each such region holds $44 \times 2$ intensity values. To create a gold standard for tissue characterization, the ROIs are labeled according to the aligned histopathology information.

To model the TeUS signals of malignant and benign ROIs, we use a time-domain representation of the ROI signals. First, we calculate the mean of the $88(44 \times 2)$ values for each ROIs. Thus, each ROI is represented by a single intensity value per time frame, and the TeUS signal associated with each ROI is represented as a sequence of 128 mean intensity values. The total number of ROIs used in our experiment is 570, of which 286 are malignant and 284 are benign. As we are interested in examining the patterns of change in echo-intensities along TeUS signals, we calculate the first-order difference along each sequence. We then discretize the values in the first-order difference series by binning their range of values into 10 equally spaced intervals. Since more than $99 \%$ of difference-values in our dataset fall in the range between -9 and 9 , this range is divided into 10 subranges (bins). Our time-domain representation thus consists of a sequence of bin ordinal-numbers, where each bin number stands for a difference-value within the bin's range.

\subsection{Tissue-characterization Framework}

To model the TeUS signals associated with the ROIs, we view the intensity values at each time frame as though they are observations generated by an unobservable stochastic process, which we model using HMMs. An HMM is defined using five elements: a set of states, an alphabet of observations, transition probabilities representing the likelihood of moving from one state to another, emission probabilities representing the likelihood of observing each alphabet symbol at each state, and initial probabilities representing the likelihood of occupying each of the state when the process starts. We train two HMMs: a malignant-tissue HMM, to model the TeUS signals of malignant ROIs, and a benign-tissue HMM to model the TeUS signals emanating from benign ROIs. HMM training is done by optimizing the model parameters, namely the transition and emission probabilities, using the Baum-Welch algorithm, which is an expectation maximization method. ${ }^{8}$

To train and test HMMs in support of tissue characterization, we use a leave-one-patient-out cross-validation. Data from ROIs of eight patients are used for training the HMMs and data from the malignant and benign ROIs of the remaining patient are used for testing. The latter are provided as the input to the malignant-tissue HMM and to the benign-tissue HMM; the output being calculated is a log likelihood score, reflecting the likelihood of each test-ROI to be generated by each of the HMMs. The log likelihood ratio is used to assign a label for the corresponding ROI; if the ratio is greater than one the ROI is labeled as malignant, otherwise it is labeled as benign. Figure 1 shows the tissue characterization framework. We assess the performance of the HMMs for differentiating between malignant and benign tissues by calculating the accuracy, sensitivity and specificity of assigning a tissue-type label (malignant vs benign) to all ROIs in the test set, and averaging these measures over the nine cross-validation runs. 


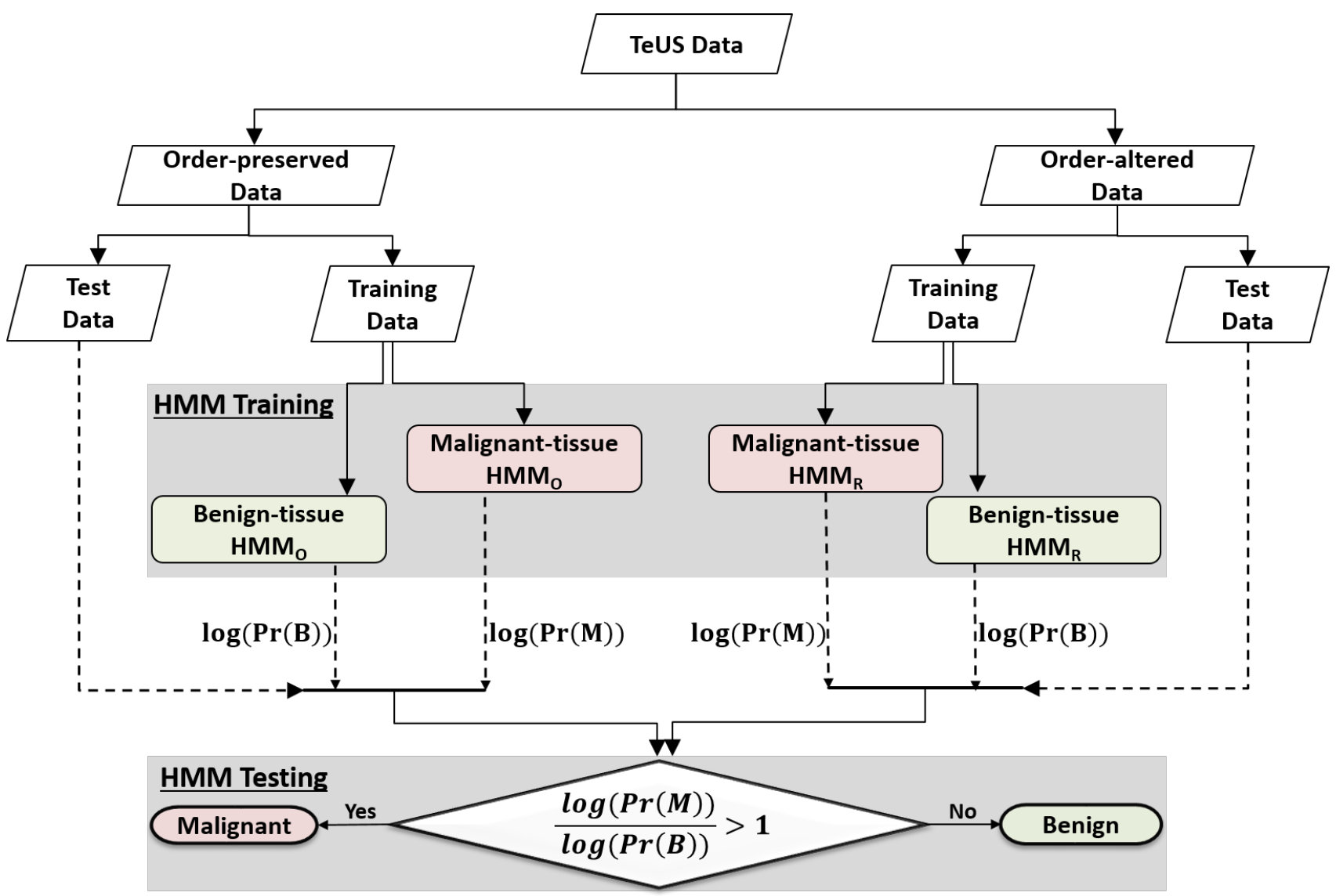

Figure 1. The tissue characterization framework using Hidden Markov Models. Order-preserved and order-altered sequences are partitioned into training and test sets. The training sets are used to train a malignant-tissue HMM and a benign-tissue HMM. We then use the learned models to produce log likelihood scores to sequences in the test set, in order to assign a label for the corresponding test ROIs.

\subsection{The impact of TeUS Temporal Order on Tissue Characterization}

To assess the impact of time-series order on the results, we generate for each ROI many instances of TeUS data where the temporal order is modified at random. We alter the order in the original data by permuting at random the order of a group of successive time-points (hereinafter referred to as the rearranged block). We use three different sizes: 32, 64 and 128 time-points. This process is illustrated in Figure 2.A. To compare the effect of the change in temporal order along various locations of the signal, we also change the starting point of rearranged block along the TeUS signal of each ROI in order to compare the effect of the time order in various locations of the signal. In total, we generate eight collections of rearranged data for each ROI: four of them have a rearranged block of size 32, starting at position 1,33, 65 or 97 along the respective sequence, other three collections have a rearranged block of size 64 starting at position 1, 33 or 65 while the last collection has a rearranged block of size 128 (all of the sequence points are included in the permutation). Figure 2.B-D show an example of an original TeUS signal from an ROI along with its respective rearranged signals.

We train malignant-tissue HMMs and benign-tissue HMMs using each of the rearranged collections and test them using the cross-validation framework described in Section 2.2. The random rearrangement is repeated 100 times and the average performance measures (accuracy, sensitivity and specificity) are reported. We compare the performance of models trained and tested over the original signals and models trained and tested over signals with temporally-rearranged blocks of different sizes. To assess the significance of the impact of sequence-reordering as a function of the size of rearranged blocks and their locations (as defined by their starting point), we employ the one tailed two-sample t-test ${ }^{12}$ with a $95 \%$ confidence interval. 

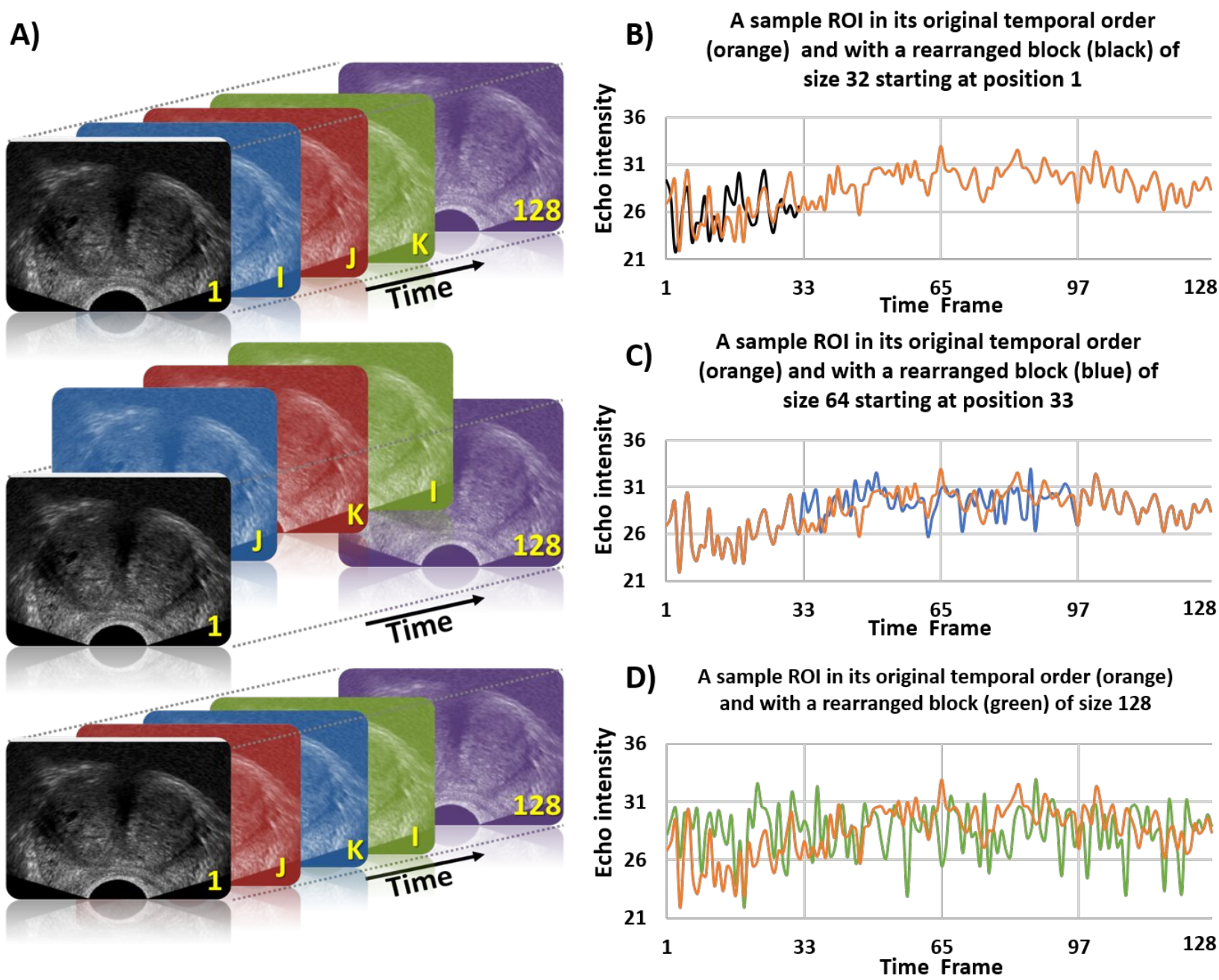

Figure 2. A) A sample sequence of TeUS illustrating the process of rearranging the time points in a block of frames of size 3. B-D) TeUS time series of a sample ROI along a time series with rearranged-blocks: a block of size 32, in black, starting at point 1 (B), a block of size 64, in blue, starting at point 33 (C), and a block of size 128 in green (D).

\section{RESULTS AND DISCUSSION}

As described above we trained two types of models: a malignant-tissue HMM and a benign-tissue HMM, using the original signals of 570 ROIs. In addition, we trained sixteen models, eight malignant-tissue HMMs and eight benign-tissue HMMs using the eight collections of rearranged ROIs. The training of models using temporallyrearranged signals was repeated 100 times for each combination of size and starting position of rearranged blocks. In each repetition, we vary the random permutation used for temporal rearrangement. The average performance was compared against the performance of the model learned from the original signals. Figure 3 shows the performance of models trained on the original signals, as well as those trained on temporally-rearranged signals, as a function of the rearranged-block size; a block-size of zero indicates the performance of HMMs trained on the original non-rearranged data sequences. The average performance measures (accuracy, sensitivity and specificity), calculated over 100 repeats of training/testing of HMMs using temporally-rearranged sequences are plotted. As shown in Figure 3, the accuracy, sensitivity and specificity all decrease as the size of the rearranged block increases. The accuracy decreases from $85 \%$ in models trained/tested using unaltered signals to $62 \%$ when models are trained/tested on temporally-modified signals with rearranged block of size 128 . This reduction in performance is statistically significant with a p-value $<<10^{-10}$. We used the two-sample t-test to calculate 

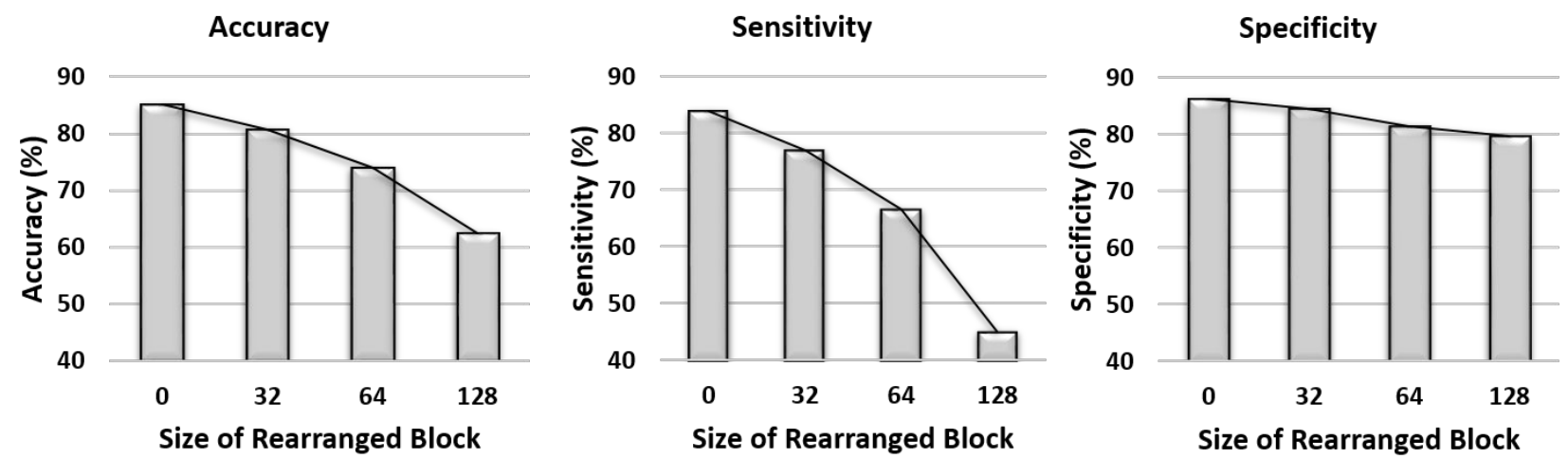

Figure 3. Accuracy, sensitivity and specificity of tissue characterization using HMMs trained and tested on TeUS time series without any temporal-rearrangement $(\mathrm{x}=0)$, compared with average performance obtained by HMMs trained and tested on TeUS time series containing rearranged-blocks of size 32, 64 and 128.
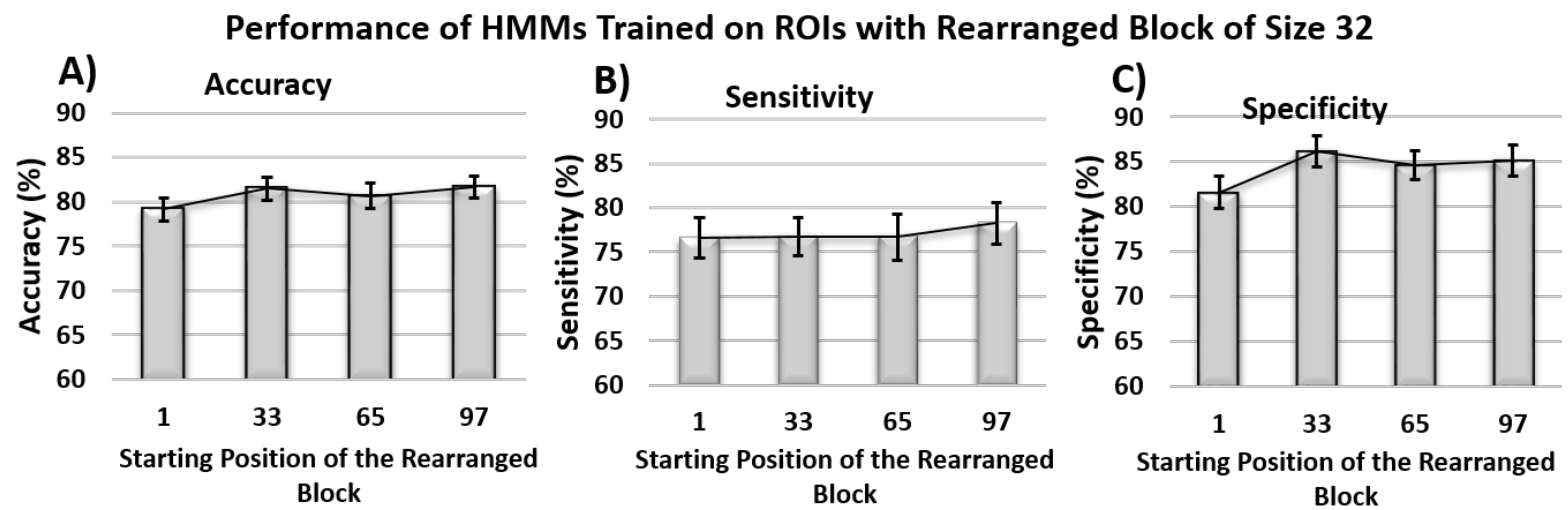

Figure 4. Accuracy, sensitivity and specificity of tissue characterization using HMMs trained and tested on TeUS time series with a rearranged block of size 32 that has four different starting points (1, 33, 65 and 97). The standard deviations of the 100 repeats are shown on top of the bars.

the statistical significance of the decrease in performance across experiments using different sizes of rearranged blocks with a confidence interval of $95 \%$. For accuracy and sensitivity, the decrease shown between each pair of adjacent bars is always statistically significant with p-value $<<10^{-2}$. As for the specificity, the degradation in performance is statistically significant except for the decrease obtained between the performance of HMMs trained/tested on original data and the performance of HMMs that modeled the signals with rearranged block of size 32. These results demonstrate that the information relayed by the temporal order of the signal is tissue specific, since rearranging the order leads to a decrease in the ability of distinguishing between TeUS signals of benign and malignant tissues. In addition, the difference between the amount of decrease in sensitivity and specificity shows that TeUS data of malignant tissues lose more information by rearranging the temporal order than TeUS data of benign tissue. In addition to the difference in performance, the topology and emission probability-distributions of the model trained on original signals and the models trained on rearranged signals are different.

We note that reduction in performance varies depending on the position of the rearranged block within the time series. Figure 4 shows the difference in accuracy, sensitivity and specificity in performance between HMMs learned from signals with a rearranged block of size 32 varying in their respective starting positions $(1,33,65$ and 97). Figure 4 shows that the impact of time rearrangement on the accuracy is higher when the rearrangement occurs toward the beginning of the signal. Using two-sample t-tests with a left tail, the accuracy and specificity of HMMs trained on signals with rearranged blocks of size 32 starting at position 1 have highly statistically significant lower values than the accuracy and sensitivity of models learned from signals with rearranged blocks 


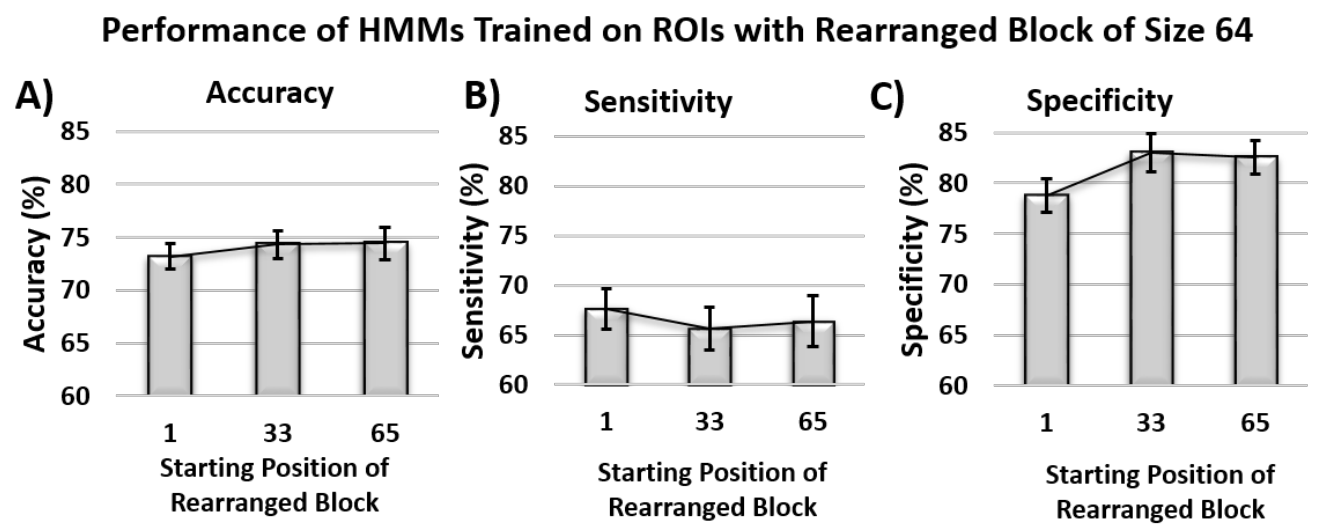

Figure 5. Accuracy, sensitivity and specificity of tissue characterization using HMMs trained and tested on TeUS time series with a rearranged block of size 64 that has three different starting points (1, 33 and 65$)$. The standard deviations of the 100 repeats are shown on top of the bars.

of size 32 starting at position 33, 65 and 97 with p-values $<<10^{-10}$. However, the sensitivity of the HMMs trained/tested on signals containing a rearranged block starting at position 1 has a statistically significantly lower value when compared with the sensitivity of models learned from signals with rearranged block starting at position 97. Figure 5 illustrates the variation in performance between HMMs trained and tested on signals whose rearranged blocks of size 64 , start at three different positions along the series (1,33 and 65). The accuracy and specificity of models learned from signals containing a rearranged block of size 64 that starts at position 1 have statistically significantly lower values than those of models trained/tested using signals with rearranged blocks starting at position 33 and 65 with p-values $<<10^{-6}$ unlike the sensitivity. The results of comparing the effect of rearranged-block's position on the performance uncover the importance of the beginning of TeUS signal on discriminating between benign and malignant tissues. Furthermore, rearranging the temporal order at the beginning of the signal resulted in altering the tissue-specific repetitive-patterns captured by the HMMs more than rearranging the order later down the signal.

\section{CONCLUSION}

We presented, for the first time, experiments aiming to directly assess the impact of temporal order of TeUS data on tissue characterization in prostate cancer, by comparing the performance of models learned from unaltered temporal signals with those learned from temporally-modified signals. We used HMMs to model prostate TeUS signals in their original temporal order as well as under a variety of temporal rearrangements. Our results show that the performance of HMMs trained to differentiate between malignant and benign prostate TeUSsignals decreases as the amount of rearrangement increases. Thus, it appears that changes to the temporal order reduces the amount of tissue-specific information relayed by the prostate-TeUS signals. Moreover, as shown in Figure 3, models learned from TeUS signals of malignant tissues are more sensitive to temporal alteration than those learned from TeUS signals of benign tissues. These findings suggest that TeUS relays a repetitive pattern of change in echo-intensities that is tissue specific, and that altering the order makes the differentiation between malignant and benign prostate tissue more difficult. As such, the temporal order is an essential characteristic of TeUS signals for tissue characterization in prostate cancer.

\section{Acknowledgments}

This work was supported in part by NSERC Discovery grants awarded to HS and to PM, NSERC and CIHR CHRP to PM, and NIH \#R56 LM011354A and NSF EAGER \#IIS-1650851 to HS. 


\section{REFERENCES}

[1] R. L. Siegel, K. D. Miller, and A. Jemal, "Cancer statistics, 2016," CA: A cancer journal for clinicians , 2015.

[2] Canadian Cancer Society, National Cancer Institute of Canada. Advisory Committee on Records, and Registries, Canadian cancer statistics, Canadian Cancer Society, 2015.

[3] H. B. Carter, P. C. Albertsen, M. J. Barry, R. Etzioni, S. J. Freedland, et al., "Early detection of prostate cancer: AUA guideline," The Journal of Urology 190(2), pp. 419-426, 2013.

[4] E. C. Serefoglu, S. Altinova, N. S. Ugras, E. Akincioglu, E. Asil, et al., "How reliable is 12-core prostate biopsy procedure in the detection of prostate cancer?," Canadian Urological Association Journal 7(5-6), pp. 293-8, 2013.

[5] E. A. Singer, A. Kaushal, B. Turkbey, A. Couvillon, P. A. Pinto, et al., "Active surveillance for prostate cancer: past, present and future," Current opinion in oncology 24(3), pp. 243-250, 2012.

[6] M. Moradi, P. Mousavi, A. H. Boag, E. E. Sauerbrei, D. R. Siemens, et al., "Augmenting detection of prostate cancer in transrectal ultrasound images using SVM and RF time series," IEEE Transactions on Biomedical Engineering 56(9), pp. 2214-2224, 2009.

[7] F. Imani, P. Abolmaesumi, E. Gibson, A. Khojaste, M. Gaed, et al., "Computer-aided prostate cancer detection using ultrasound RF time series: In vivo feasibility study," IEEE Transactions on Medical Imaging 34(11), pp. 2248-2257, 2015.

[8] L. R. Rabiner, "A tutorial on hidden Markov models and selected applications in speech recognition," Proceedings of the IEEE 77(2), pp. 257-286, 1989.

[9] N. N. Bitar and C. Y. Espy-Wilson, "Knowledge-based parameters for HMM speech recognition," in Proceedings of the IEEE International Conference on Acoustics, Speech, and Signal Processing, 1, pp. 29-32, IEEE, 1996.

[10] S. R. Eddy , "Multiple alignment using hidden markov models.," in Proceedings of the International Systems on Molecular Biology, 3, pp. 114-120, 1995.

[11] L. Nahlawi, F. Imani, M. Gaed, J.A Gomez, M. Moussa, et al., "Prostate cancer: Improved tissue characterization by temporal modeling of radio-frequency ultrasound echo data," in Proceedings of the International Conference on Medical Image Computing and Computer Assisted Intervention., 2016.

[12] C. Stein, "A two-sample test for a linear hypothesis whose power is independent of the variance," The Annals of Mathematical Statistics 16(3), pp. 243-258, 1945. 